\title{
Perioperative Management of Staged Palliation Surgery for Functional Univentricle Hearts: A Literature Review
}

\author{
Moira Setiawan ${ }^{1}$, Suprayitno Wardoyo $^{1}$, Dhama Shinta Susanti ${ }^{1}$, William Makdinata ${ }^{1}$, and \\ * Ardiansyah ${ }^{1}$ \\ ${ }^{1}$ Universitas Indonesia Fakultas Kedokteran
}

November 30, 2021

\begin{abstract}
Around 3-4 children born with congenital heart diseases have univentricular hearts, where the prognosis of univentricular hearts is poor in the past, with a survival rate of less than $50 \%$ during the first year and $10 \%$ during the first ten years of life. Based on a literature search, current advances in perioperative management of neonates with complex congenital heart diseases have increased their survival rate by $85 \%$. To aid cardiothoracic surgeons worldwide, this comprehensive literature review will focus on the perioperative management of staged palliation surgery for functional univentricular hearts, considering current trends as well as how we do it in our centre. Our review article specifially discusses perioperative strategies regarding surgical considerations, current techniques, to deal with overshunting and undershunting during the first stage of palliation surgery. This article also gives an overview on when a patient is suitable to go through with the next stage of the procedure, which is the implementation of a bidirectional cavopulmonary shunt or the Hemi-Fontan procedure. Lastly, this article gives a comprehensive approach regarding perioperative strategies of the Fontan procedure, which include patient criteria, current surgical techniques, postoperative management, as well as the use of anticoagulants after the Fontan procedure.
\end{abstract}

Perioperative Management of Staged Palliation Surgery for Functional Univentricle Hearts: A Literature Review

Suprayitno Wardoyo ${ }^{1}$, Dhama Shinta Susanti ${ }^{1}$, William Makdinata ${ }^{1}$, Ardiansyah $^{1}$, Moira Setiawan ${ }^{2}$

Division of Pediatric and Congenital Heart Surgery, Department of Surgery, Cipto Mangunkusumo Hospital, Faculty of Medicine, Universitas Indonesia, Jakarta, Indonesia

Faculty of Medicine, Universitas Indonesia, Jakarta, Indonesia

\section{*Corresponding author}

Moira Setiawan

Faculty of Medicine, Universitas Indonesia, 06 Salemba Raya St., Central Jakarta, 10430, Capital City of Jakarta, Indonesia

Telephone number: +628111195159

E-mail: moirasetiawan@gmail.com

Data Availability: The data associated with the paper are not publicly available but are available from the corresponding author on reasonable request.

Funding: None

Conflict of Interest: None 
International Review Board approval: N/A Informed Consent: N/A

Clinical Trial Registration: N/A

\begin{abstract}
Around 3-4 children born with congenital heart diseases have univentricular hearts, where the prognosis of univentricular hearts is poor in the past, with a survival rate of less than $50 \%$ during the first year and $10 \%$ during the first ten years of life. Based on a literature search, current advances in perioperative management of neonates with complex congenital heart diseases have increased their survival rate by $85 \%$. To aid cardiothoracic surgeons worldwide, this comprehensive literature review will focus on the perioperative management of staged palliation surgery for functional univentricular hearts, considering current trends as well as how we do it in our centre. Our review article specifially discusses perioperative strategies regarding surgical considerations, current techniques, to deal with overshunting and undershunting during the first stage of palliation surgery. This article also gives an overview on when a patient is suitable to go through with the next stage of the procedure, which is the implementation of a bidirectional cavopulmonary shunt or the Hemi-Fontan procedure. Lastly, this article gives a comprehensive approach regarding perioperative strategies of the Fontan procedure, which include patient criteria, current surgical techniques, postoperative management, as well as the use of anticoagulants after the Fontan procedure.
\end{abstract}

Keywords: fontan procedure, perioperative management, staged palliation, univentricular hearts

\title{
Introduction
}

Congenital heart diseases (CHD) are the most frequent congenital defect found in neonates, accounting for nearly one-third of major congenital anomalies. ${ }^{1}$ CHD that cannot be surgically reconstructed to achieve normal anatomic conditions of biventricular circulation are often described as functional univentricular hearts (UVH). It is reported that the incidence of functional UVH is 3 to 4 per 100 children born with CHD. ${ }^{2}$ The natural history of functional UVH is poor in the past, with a reported survival rate of less than $50 \%$ at one year and $10 \%$ at ten years. Advances in perioperative management for neonates with complex CHD have increased survival rates by more than $85 \% .^{3,4}$

The functional univentricular heart can result from a variety of anatomic lesions. Regardless of the etiology, newborns with these lesions mix systemic and pulmonary venous blood. Perioperative management is becoming as important in patients with UVHs as preoperative patient management focuses on the balance of competing circulations, where systemic and pulmonary circulations exist in parallel rather than in series, as in normal circulation. Thus, most patients will require surgical intervention to provide unobstructed systemic blood flow (Qs) and restrictive pulmonary blood flow (Qp). ${ }^{5}$ Intraoperative management focuses on selecting ideal candidates for each staged palliation surgical procedure such as pulmonary artery size and pulmonary artery resistance index, while postoperative patient management focuses on optimizing systemic output, respiratory status, and mitigating the effects of cardiopulmonary bypass. ${ }^{5}$

\section{First Stage of Univentricular Hearts}

\section{Blalock-Taussig-Thomas Shunt (BTTS)}

BTTS represents the first stage of univentricular palliation surgery in patients with restricted pulmonary blood flow. This procedure allows the growth of pulmonary arteries, where it regulates pulmonary blood flow to the lungs until the size of the pulmonary artery is suitable for the second stage of univentricular palliation surgery. The classic BTTS is a direct anastomosis between the transected subclavian artery (or the innominate artery) and the pulmonary artery (Figure 1 ). It requires extensive surgical dissection where it sacrifices the subclavian artery. ${ }^{6}$ The main disadvantages of classic BTTS include long operative dissection time, phrenic nerve injury, technical difficulties during the takedown, as well as possible arm ischemia. In 1975, this technique was modified by Marc R. de Leval and his colleagues, where a polytetrafluoroethylene (PTFE) graft was used to interpose between the subclavian artery and the ipsilateral pulmonary artery (Figure $\mathbf{2}$ ). Since this modification, the procedure is now more popularly known as the modified 
BTTS. PTFE conduits are considered ideal compared to Dacron because they have smaller pore sizes that limit ingrowth of tissue, yet allowing for fibroblastic incorporation to bind the conduit to its surrounding structures. ${ }^{7}$

To maximize postoperative outcomes of the modified BBTS, a few things regarding perioperative management such as surgical consideration as well as strategies to deal with over-shunting and under-shunting must be considered. Surgical considerations include the approach to apply the modified BTTS. An approach through median sternotomy gives the surgeon exposure to the right subclavian artery. It will also expand the surgical field and allows the surgeon to initiate cardiopulmonary bypass if needed. The approach through sternotomy is correlated to the extended use of mechanical ventilatory support, the length of stay in the intensive care unit and hospital, as well as a higher mortality rate. Meanwhile, the thoracotomy approach provides ease in creating a proximal anastomosis beyond the bifurcation of the innominate artery. The thoracotomy approach will lead to the need for a more extended graft. The diameter of the graft becomes less critical in estimating shunt resistance so that the flow can be a problem. A longitudinal arteriotomy incision in the subclavian and pulmonary arteries provides a wider circumferential area of the anastomosis for a larger shunt size. This technique proves to be more beneficial for hypoplastic pulmonary arteries. Pulmonary artery distortion at the anastomotic site is also less common with the longitudinal incision technique. Using a smaller needle size of $8 / 0$ polypropylene will help provided better anastomosis and less bleeding from the needle hole. ${ }^{8}$

The basic principle to deal with over-shunting and under-shunting include balancing Qp:Qs by decreasing systemic vascular resistance (SVR) and increasing pulmonary vascular resistance (PVR) in modified BTTS to achieve an oxygen saturation of 70-85\%. Strategies to increase the PVR include reducing the fraction of inspired oxygen (FiO2) to 0.21, avoidance of hyperventilation with targeted permissive hypercapnia, administration of high PEEP (PEEP 6-8), and maintaining blood $\mathrm{pH}$ 7.35-7.40. Evaluation of lactic acid and arterial blood gas may be done every 4-6 hours. Some inodilator are practical and can be used to decrease SVR. Dobutamine, milrinone or levosimendan may be considered while maintaining a diastolic blood pressure of over $25 \mathrm{mmHg}$. Administration of nitroglycerin can be considered if blood pressure is still high after using the inodilator.

After establishing the signs of under-shunting (oxygen saturation $<70 \%$, despite being given $\mathrm{FiO} 2>0.60$ ), we must first exclude the cause of under-shunting, especially shunt thrombosis where urgent echocardiography is needed to evaluate shunt patency. Respiratory system failure, such as displaced endotracheal tube, obstructed endotracheal tube, pneumothorax, and failure of hemodynamic support and equipment may also cause under-shunting. If those mentioned above are already excluded, strategic management include increasing FiO2 to achieve an oxygen saturation of 70-85\%, avoid acidosis, and keeping blood pH around 7.40 to 7.45. Adequate volume status must be ensured by titrating fluid slowly according to the patient's response with $5 \mathrm{ml} \mathrm{5 \%}$ albumin. If the blood pressure is low, consider starting vasoconstrictors, such as norepinephrine or epinephrine. In addition, a pulmonary vasodilator (sildenafil and inhaled nitric oxide) may be needed to decrease PVR. ${ }^{8}$

\section{Pulmonary Artery Banding (PAB)}

PAB represents the first stage of univentricular palliation surgery in patients with unrestricted pulmonary blood flow. PAB aims to decrease pulmonary artery pressure and pulmonary vascular resistance to levels suitable for future univentricular palliation surgery. The golden period for performing PAB is considered to be 2 to 4 weeks of age. By this time, the neonatal pulmonary vascular resistance decreases, allowing for a tighter band. ${ }^{9}$

A median sternotomy approach was used for PAB. We use Mersilene tape with $5 \mathrm{~mm}$ width (Ethicon Inc., Somerville, NJ, USA) to band the pulmonary artery (Figure 3). To prevent migration, the tape was fixated with two 6/0 Polypropylene stitches. As a guide, the Trusler formulae (for univentricle hearts: 22 $\mathrm{mm}+$ weight in $\mathrm{kg}$ ) are used as the first steps, then finer adjustments being made by systemic $\mathrm{O} 2$ saturation (75-85\%, with inspired oxygen fraction 0.50) and distal PAP (Target distal PAP $50 \%$ or the optimal mean 
PAP $<15 \mathrm{mmHg}){ }^{9}$

\section{Second Stage of Univentricular Hearts}

The goal of the second-stage procedure of UVH is to divert systemic venous blood from the superior vena cava directly into the pulmonary vascular bed (Figure 4), allowing for effective pulmonary blood flow while reducing volume load on the single ventricle to allow for favourable ventricular remodelling, which improves the patients' outcomes. ${ }^{10}$

The bidirectional cavopulmonary shunt (BPCS) or Hemi-Fontan procedure is usually performed when the pulmonary arteries have grown adequately to allow adequate pulmonary blood flow with low PVR, which is usually between 2 to 6 months. ${ }^{11}$ In cases where the modified BTTS or PAB is performed first, reassessment to measure pulmonary artery size adequacy and pulmonary artery resistance index will be carried out 6 to 12 months later using cardiac catheterization or multi-slice computed tomography (MSCT).

The selection of the second-stage shunting procedure determines the technique utilized for the completion of the Fontan procedure. When a BCPS is used, an extracardiac completion Fontan is performed, as there is no point in reconnecting the superior vena cava to the right atrium after a BCPS. Patients with Hemi-Fontan modifications have extremely relevant anatomy for completion by the lateral tunnel Fontan operation, thus preferably used in these patients. ${ }^{12}$ The criteria for conducting BCPS or the Hemi-Fontan procedure at our centre include a mean pulmonary artery pressure of less than $18 \mathrm{mmHg}$ and a pulmonary artery resistance index of less than Wood units $/ \mathbf{m}^{\mathbf{2}}$, with confluent pulmonary arteries and making sure the pulmonary artery size is according to the half size of the patient, which depends on body weight. Usually, in cases of UVH in BCPS, atrial septectomy will be performed if a restrictive atrial septal defect is suspected.

Postoperative management include positioning of the patient, where we usually position the patient in a semi-Fowler position to improve blood flow from the upper body to the right atrium. Early extubation helps improve pulmonary blood flow and systemic oxygen delivery as well as avoid needless sedation to improve spontaneous breathing. Modest hypercarbia of pCO2 $\pm 45 \mathrm{mmHg}$ is acceptable as it enhances cerebral vasodilation and reduces the superior vena cava pressure. The target of saturation oxygen for BCPS/HemiFontan patients is $85 \%$. We typically give inotropics such as dobutamine to improve stroke volume in patients with poor contractility. Pulmonary vasodilators such as milrinone or oral sildenafil (if the patient can tolerate oral feeding) may be given. Patients with bilateral BCPS or concomitant reconstruction of pulmonary artery branches are usually given $5 \mathrm{mg} / \mathrm{kg}$ of acetylsalicylic acid in our centre.

\section{Third Stage of Univentricle Hearts (Fontan Procedure)}

The Fontan procedure is the third and last stage of the palliation procedure for UVH. The procedure is performed to divert the remaining systemic venous blood from the inferior vena cava to the pulmonary artery (Figure 5 ). This procedure is usually performed at around 1 to 5 years of age when restriction of patient activity is becoming problematic and size of the pulmonary arteries are sufficient to allow for a low PVR. ${ }^{13}$ The classic Fontan technique include creating an atriopulmonary anastomosis is made by isolating the right atrial chamber by closing the atrial septal defect and connecting the hypoplastic tricuspid valve to the inferior vena cava. Afterwards, the right atrial appendage would be anastomosed to the right pulmonary artery. It was later understood that better streaming the blood flow in the systemic venous pathway to the lungs could improve the patients' hemodynamics. By doing so, complications related to progressive atrial dilation could be avoided. The operation was subsequently modified to what is known as the lateral tunnel technique. The right atrium was baffled with an intra-atrial patch, and the superior vena cava was sutured directly to the right pulmonary artery. ${ }^{13}$ The most recent modification of the Fontan procedure consisted of replacing the intra-atrial routing of the venous blood by inserting an extracardiac conduit between the inferior vena cava and the right pulmonary artery. ${ }^{14}$ The advantages of using an extracardiac conduit include avoiding multiple suture lines in the atrium that might serve as a substrate for reentrant tachycardias. ${ }^{15}$

Our criteria in selecting candidates that can be safely staged and are eligible for the Fontan procedure to ensure a high probability of success include a mean pulmonary artery pressure of less than $15 \mathrm{mmHg}$, 
pulmonary artery resistance index of less than 4 Wood units $/ \mathrm{m}^{2}$, confluent pulmonary arteries with the diameter of the branches according to the patient's bodyweight in the half size, systemic ventricular enddiastolic pressure of less than $15 \mathrm{mmHg}$, the absence of severe atrioventricular valve regurgitation unless it can be repaired or replaced, good systemic ventricular function, and the patient's age should be more than three years.

The patient is usually positioned in a semi-Fowler position after the procedure to improve blood flow from the upper body to the right atrium. Early ambulation after surgery also increases venous return and cardiac output. Early extubation will also improve pulmonary blood flow and systemic oxygen delivery, provided that spontaneous breathing provides enough oxygenation and $\mathrm{CO}_{2}$ removal. ${ }^{16}$ The optimal target for oxygen saturation after the Fontan Procedure is 95\%. Low positive end-expiratory pressure and low mean airway pressure are ideal if the patient still needs ventilator support.

Regarding anticoagulant management, we start the patient on heparin as soon as possible. If there is no evidence of bleeding, heparin is administrated at an initial dose of $5-10 \mathrm{IU} / \mathrm{kg} / \mathrm{hour}$ and titrated until the therapeutic level is achieved, indicated by a prothrombotic time of $60-80$ seconds. In addition, we usually start the patient on $0,2 \mathrm{mg} / \mathrm{kg} /$ day of warfarin after extubation, where it is titrated every three days until therapeutic level is achieved, indicated by an international normalized ratio (INR) of 2.0-3.0.

\section{Reference}

1. Abid D, Elloumi A, Abid L, Mallek S, Aloulou H, Chabchoub I, et al. Congenital heart disease in 37,294 births in Tunisia: birth prevalence and mortality rate. Cardiol Young. 2014;24(5):866-71.

2. Idorn L, Olsen M, Jensen AS, Juul K, Reimers JI, Sorensen K, et al. Univentricular hearts in Denmark 1977 to 2009: incidence and survival. Int J Cardiol. 2013;167(4):1311-6.

3. Medoff-Cooper B, Irving SY, Marino BS, Garcia-Espana JF, Ravishankar C, Bird GL, et al. Weight change in infants with a functionally univentricular heart: from surgical intervention to hospital discharge. Cardiol Young. 2011;21(2):136-44.

4. Chamaidi A, Gatzoulis MA. Heart disease and pregnancy. Hellenic J Cardiol. 2006;47(5):275-91.

5. Villa CR, Marino BS, Jacobs JP, Cooper DS. Intensive care and perioperative management of neonates with functionally univentricular hearts. World J Pediatr Congenit Heart Surg. 2012;3(3):359-63.

6. Ullom RL, Sade RM, Crawford FA, Jr., Ross BA, Spinale F. The Blalock-Taussig shunt in infants: standard versus modified. Ann Thorac Surg. 1987;44(5):539-43.

7. Gazzaniga AB, Elliott MP, Sperling DR, Dietrick WR, Eiseman JT, McRae DM, et al. Microporous expanded polytetrafluoroethylene arterial prosthesis for construction of aortopulmonary shunts: experimental and clinical results. Ann Thorac Surg. 1976;21(4):322-

8. Wardoyo S, Makdinata W, Wijayanto MA. Perioperative strategy to minimize mortality in neonatal modified Blalock-Taussig-Thomas Shunt: A literature review. Cirugía Cardiovascular. 2021. Available from: https://doi.org/10.1016/j.circv.2021.04.002

9. Sasikumar N, Ramanan S, Rema KM, Subramanyan R, Kumar RS, Cherian KM. Pulmonary artery banding for univentricular heart beyond the neonatal period. Asian Cardiovasc Thorac Ann. 2014;22(6):6606 .

10. Lee TM, Aiyagari R, Hirsch JC, Ohye RG, Bove EL, Devaney EJ. Risk factor analysis for second-stage palliation of single ventricle anatomy. Ann Thorac Surg. 2012;93(2):614-8; discussion 9.

11. Hirsch-Romano JC, Bove EL, Si M-S, Ohye RG. Modified Hemi-Fontan Procedure. Operative Techniques in Thoracic and Cardiovascular Surgery. 2013;18(2):117-23.

12. Spray TL. Hemi-Fontan Procedure. Operative Techniques in Thoracic and Cardiovascular Surgery. 2013;18(2):124-37. 
13. d'Udekem Y, Iyengar AJ, Cochrane AD, Grigg LE, Ramsay JM, Wheaton GR, et al. The Fontan procedure: contemporary techniques have improved long-term outcomes. Circulation. 2007;116(11 Suppl):I157-64.

14. Norwood WI, Jacobs ML. Fontan's procedure in two stages. The American Journal of Surgery. 1993;166(5):548-51.

15. Jaquiss R, Imamura M. Single ventricle physiology: surgical options, indications and outcomes. Curr Opin Cardiol. 2009;24(2):113-8.

16. Mittnacht AJC. Pro: Early Extubation Following Surgery for Congenital Heart Disease. Journal of Cardiothoracic and Vascular Anesthesia. 2011;25(5):874-6.

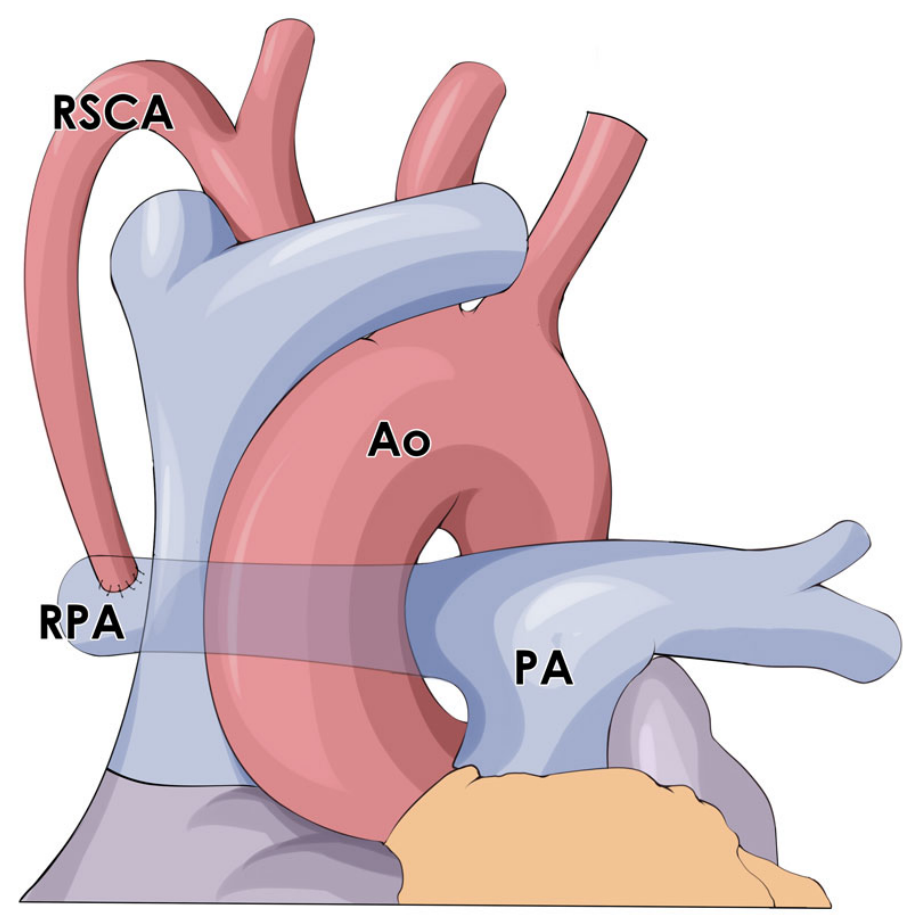

Figure 1. Classic Blalock-Taussig's shunt.

AO: aorta; PA: pulmonary artery; RPA: right pulmonary artery 


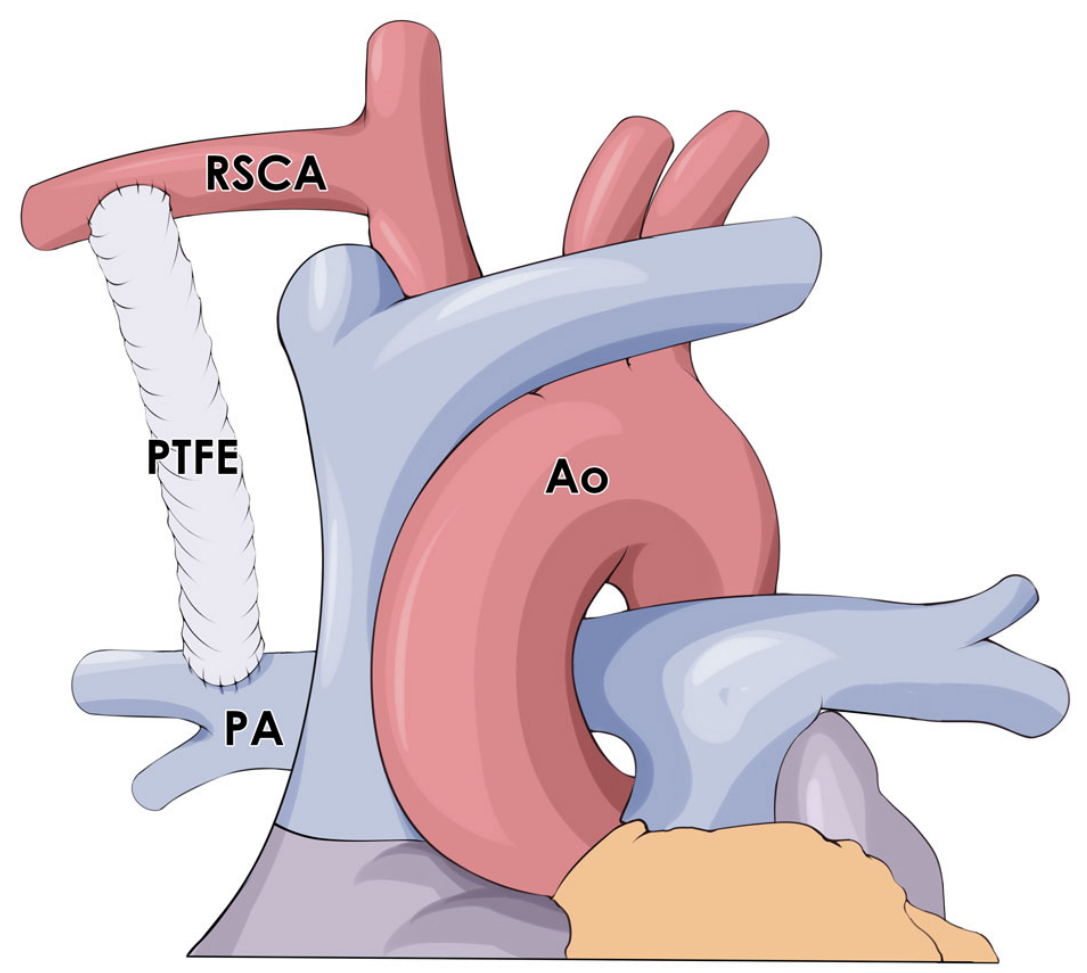

Figure 2 . Modified Blalock-Taussig's shunt.

AO: aorta; PA: pulmonary artery; PTFE: polytetrafluoroethene; RSCA: right subclavian artery 


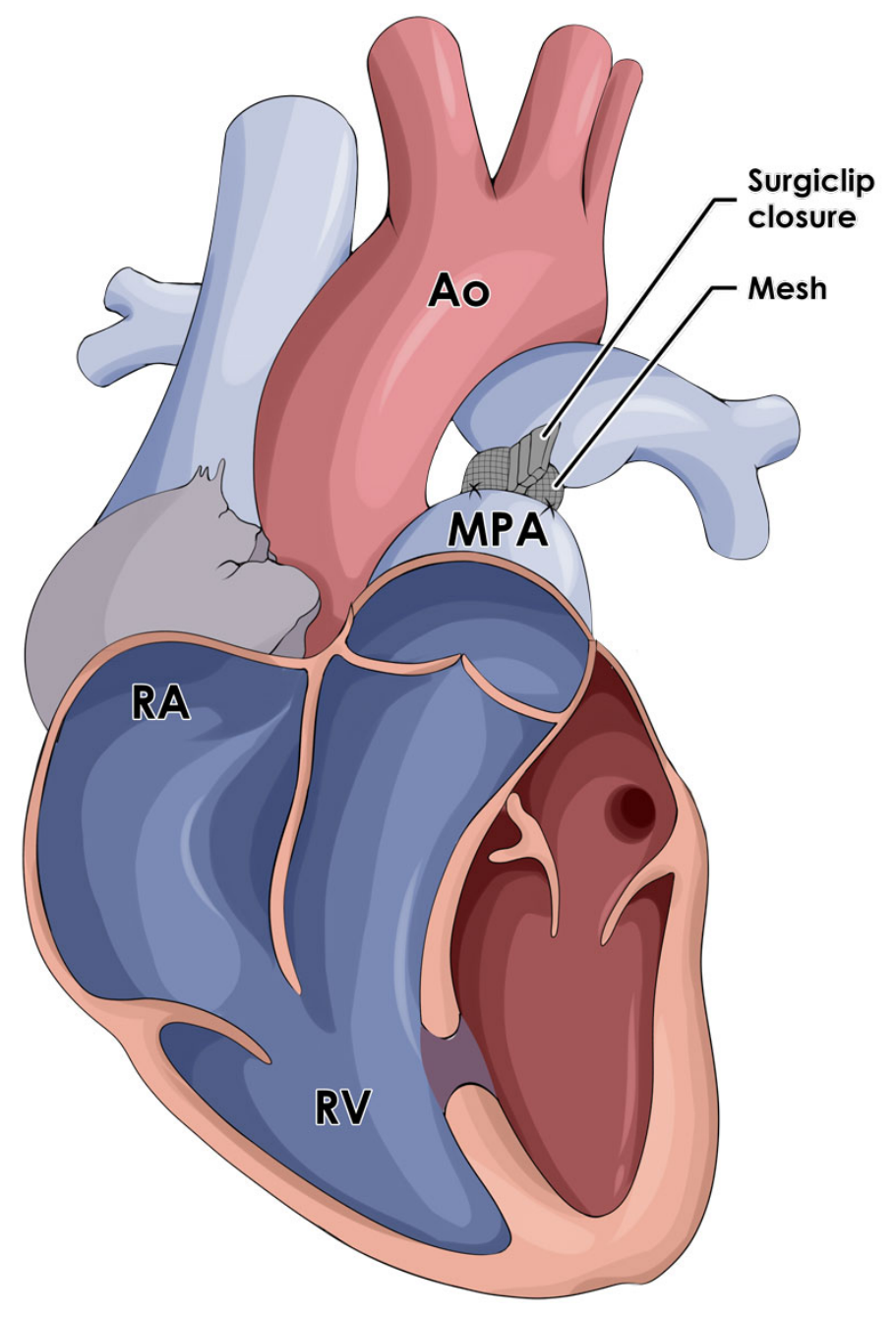

Figure 3 . Pulmonary Artery Banding

AO: aorta; PA: pulmonaryartery; MPA: main pulmonary artery

RA: right atrium; RV: right ventricle 


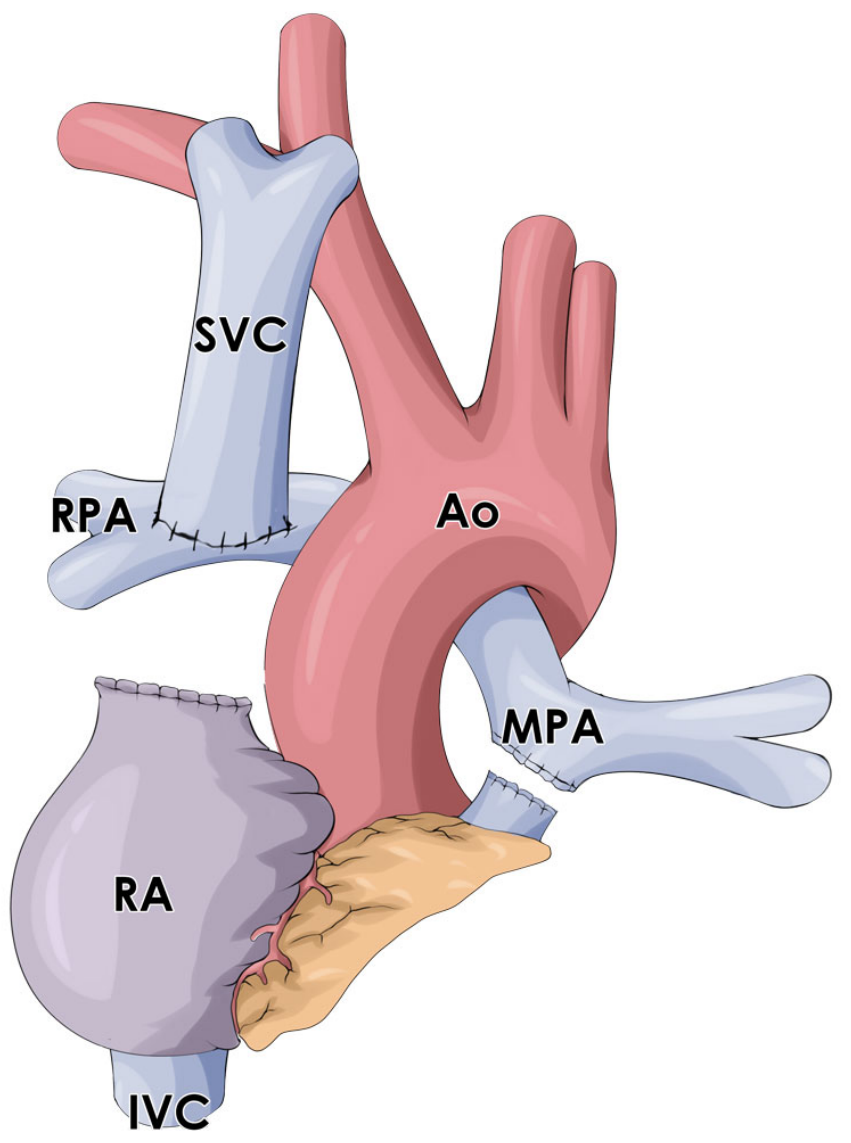

Figure 4 . The Bidirectional Glenn Shunt

AO: aorta; PA: pulmonaryartery; RPA: right pulmonary artery

MPA: main pulmonary artery; RA: right atrium; IVC: inferior vena cava
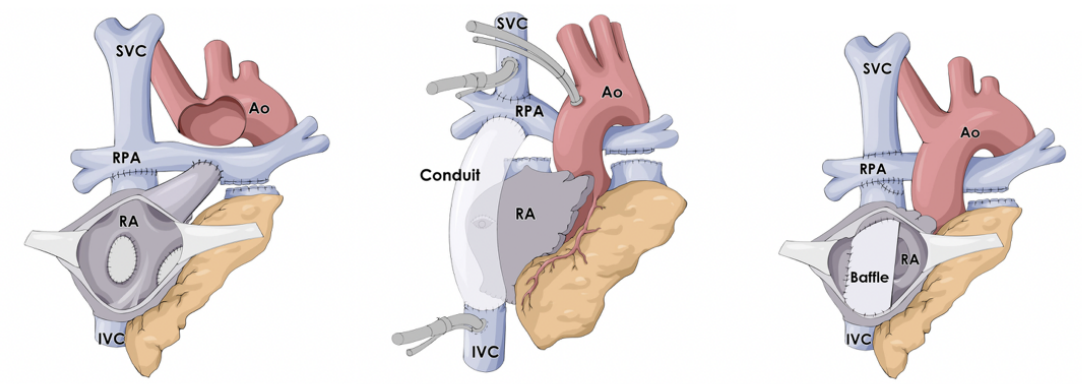

Figure 5. (A) Classical Fontan, (B) extracardiac conduit Fontan and (C) Lateral tunnel Fontan. 